J. Clin. Chem. Clin. Biochem.

Vol. 17, 1979, pp. 115-121

\title{
Eine einfache gaschromatographische Methode zur Bestimmung von Schlafmitteln im Serum
}

\author{
Von W. R. Külpmann ${ }^{1}$ ) \\ Institut für Klinische Chemie (geschäftsf. Direktor Prof. Dr. Dr. J. Büttner) Medizinische Hochschule Hannover
}

(Eingegangen am 24. Juli/4. Oktọer 1978)

Zusammenfassung: Es wird gezeigt, daß sich in dem nach Külpmann ((1979), diese Z. 17, 89-96) hergestellten Serumextrakt nicht nur Barbiturate, sondern auch andere Schlafmittel gaschromatographisch bestimmen lassen: Carbromal, 2,2-Diethylallylacetamid, Ethinamat, Glutethimid, Methyprylon und Pyrithyldion. Methaqualon wird qualitativ nachgewiesen. Der Variationskoeffizient für die Präzision in der Serie schwankt - je nach Substanz zwischen 2,1 und 8,5\%, die Wiederfindung zwischen 76 und 92\%; die Nachweisgrenze beträgt 1,6 bis $4,6 \mu \mathrm{mol} / 1$. Die Spezifität wird überprüft durch Vergleich:

1. der Analysen von Serumproben von Vergiftungen vor und nach zusätzlicher dünnschichtchromatographischer Reinigung des Extraktes,

2. mit den Retentionszeiten von etwa 100 Arzneimitteln bei gaschromatographischer Analyse an den 3 verwendeten stationären Phasen. Die Methode erlaubt den Ausschluß bzw. Nachweis und quantitative Bestimmung von 18 barbiturat- und 7 nichtbarbiturathaltigen Schlafmitteln in 1 bis 2 Stunden.

\section{A simple gas chromatographic method for the determination of hypnotics in the serum}

Summary: In the extract of the serum, prepared as described by Külpmann (1979) (this J. 17, 89-96), other hypnotics, in addition to the barbiturates can be determined by gas chromatography; these are: carbromal, 2,2diethylallylacetamide, ethinamate, glutethimide, methyprylone and pyrithyldione. Methaqualone can be detected qualitatively. The coefficient of variation for the precision in the series is dependent on the hypnotic investigated and ranges from 2.1 to $8.5 \%$, the recovery from 76 to $92 \%$; the detection limit is estimated to be 1.6 up to $4.6 \mu \mathrm{mol} / 1$. The specificity was proved by comparison

1) of analyses of sera from poisoned patients or animals before and after the additional purification of the extract by thin-layer chromatography,

2) with the retention times of about 100 drugs under the gas chromatographic conditions that were used. The method allows the determination of 18 barbiturates and 7 non-barbiturates within one to two hours.

\section{Einleitung}

Noch in den Jahren 1954-56 wurden 70\% aller Patienten aügrund der Einnahme einer Úberdoșis eines barbiturathaltigen Schlafmittels in das Vergiftungszentrum Kopenhagens eingeliefert (2). Seitdem hat sich die Vergiftungsszene wesentlich gewandelt. In der Bundesrepublik Deutschland dürfte nach Erhebungen von $v$. Clarmann (3) der Barbituratanteil an den Hypnoticasuiciden nur noch $20-25 \%$ ausmachen, die übrigen $75-80 \%$ entfallen auf die Gruppe der Nichtbarbiturate: Bromureide, insbesondere Carbromal, 2,2-Diethylallylacetamid,

1) Ausziugsweisge vorgetragen auf der Tagung Biochemische Analy tik 1978 und Analytika 1978, 18.-21. April 1978, München.
Ethinamat, Glutethimid, Methaqualon, Methyprylon und Pyrithyldion. Von diesen Substanzen besaß in den letzten Jahren Carbromal eine überragende Bedeutung, da fast die Hälfte der Schlafmittelvergiftungen auf dieses Hypnoticum entfielen. Wie schon bei der Einführung der Rezeptpflicht für z. B. Glutethimid oder Methyprylon beobachtet, dürfte die Einführung der Verschreibungspflicht für Bromureide zu einer raschen Verminderung ihrer toxikologischen Bedeutung führen. Es ist zu erwarten, daß stattdessen andere, nicht rezeptpflichtige Schlafmittel in vermehrtem Umfang mißbräuchlich eingenommen werden. Wegen der bevorstehenden Umschichtung bei den Schlafmittelintoxikationen, der häufigen gleichzeitigen Verwendung von verschiedenen Arzneistoffen und der Bevorzugung von Mischpräpara- 
ten sollte eine Methode aufgebaut werden, die möglichst in einem Ansatz alle Hypnotica im Serum zu identifizieren und quantitativ zu bestimmen gestattet. Außer auf die unabdingbare Zuverlässigkeit sollte auf die Praktikabilität des Verfahrens geachtet werden, um zu gewährleisten, daß rund um die Uhr auch mit weniger geschultem Personal in 1-2 Stunden die Fragen des behandelnden Arztes beantwortet werden können: Liegt eine Schlafmittelvergiftung vor? Welche Hypnotica wurden eingenommen? Wie hoch ist ihre Konzentration im Serum?

\section{Material und Methodik}

Material

Carbromal (2-Brom-2-ethyl-butyryl-harnstoff) relative Molekülmasse $\left(M_{r}\right) 237,1$ (Bayer, Leverkusen)

2,2-Diethylallylacetamid, $M_{r} 155,2$ (Much, Bad Soden)

Ethinamat (1-Ethinyl-cyclohexyl-carbamat), $M_{\mathrm{r}}$ 167,2 (Asche, Hamburg)

Glutethimid (2,6-Dioxo-3-ethyl-3-phenyl-piperidin), $\mathrm{M}_{\mathrm{r}} 217,3$ (Ciba Wehr)

Hexobarbital (5-(Cyclohex-1-en-yl)-1,5-dimethyl-barbitursäure), $M_{r}$ 236,3 (Bayer, Leverkusen)

Methaqualon (2-Methyl-3-(o-tolyl)-(3H)-chinazolin-4-on), $\mathrm{M}_{\mathrm{r}}$ 250,4 (Cascan, Wiesbaden-Schierstein)

Methyprylon (2,4-Dioxo-3,3-diethyl-5-methyl-piperidin), $\mathrm{M}_{\mathrm{r}}$ 183,3 (Hoffmann-La Roche, Grenzach-Wyhlen)

Pyrithyldion (2,4-Dioxo-3,3-diethyl-tetrahydropyridin), $\mathrm{M}_{\mathbf{r}}$ 167,2 (Hoffmann-La Roche, Grenzach-Wyhlen)

Die Reinsubstanzen wurden von den Firmen kostenlos zur Verfuigung gestellt. Sie wurden einzeln in Ethylacetat/Eisessig $(100 \mathrm{ml}+1 \mathrm{ml})$ gelöst. Die Konzentration betrug $1 \mathrm{~g} / \mathrm{l}$. Die Lösungen sind bei $4^{\circ} \mathrm{C}$ mindestens drei Monate haltbar.

$\left[2-{ }^{14} \mathrm{C}\right]$ Hexobarbital wurde von NEN (Dreieichenhain) bezogen; spezifische Aktivität $317,5 \mathrm{GBq} / \mathrm{mol}(8,58 \mathrm{mCi} / \mathrm{mmol})$. Das Barbiturat wurde in dreimonatigen Abständen radiodünnschichtchromatographisch auf Reinheit untersucht. Die Gebrauchslösung en thielt 7,4 MBq/1 $(200 \mu \mathrm{Ci} / 1)$ Ethanol.

\section{Geräte und Zubehör}

Die gaschromatographische Analyse wurde durchgeführt mit Geräten der Fa. Varian (Darmstadt), Modell 1400 und 2800, beide ausgerüstet mit Flammenionisationsdetektor.

Als Trägergas wurde nachgereinigter Stickstoff ( $35 \mathrm{ml} / \mathrm{min}$ ) benutzt, als Brenngas nachgereinigter Wasserstoff und synthe tische Luft. Die silikorisierten Glassäulen waren gefüllt mit:

1. 3\% OV-101 auf Chromosorb WHP 100-120 mesh

2. 3\% SP 2250 DA auf Chromosorb WHP 100-120 mesh

3. 3\% CDMS auf Chromosorb WHP 100-120 mesh

Die Phasen wurden von Supelco (Bellefonte, Pa. U.S.A.) bezogen.

\section{Gaschromatographische Bedingungen}

Säule 1: Einlaß: $170^{\circ} \mathrm{C}$, Säule: $150^{\circ} \mathrm{C}$ und Detektor: $185^{\circ} \mathrm{C}$. Säule 2: Einlaß: $220^{\circ} \mathrm{C}$, Säule: $190^{\circ} \mathrm{C}$ und Detektor: $220^{\circ}$.C. Säule 3: Einlaß: $250^{\circ} \mathrm{C}$, Säule: $220^{\circ} \mathrm{C}$ und Detektor: $250^{\circ} \mathrm{C}$.

Der Säuleneingang wird nicht mit silikonisierter Watte verschlossen.

Die quantitative Bestimmung von Carbromal, 2,2-Diethylallylace tamid, Ethinamat und Pyrithyldion erfolgt an Säule 1, von Glutethimid an Säule 2 und Methyprylon an Säule 3. Methaqualon wird qualitativ an Säule 2 und 3 nachgewiesen.

Die übrigen Angaben entsprechen sinngemäß 1. c. (1).

\section{Methodik}

Die Aufarbeitung der Probe erfolgt wie beschrieben (1): Die Serumprobe wird mit gesättigter $\left(\mathrm{NH}_{4}\right)_{2} \mathrm{SO}_{4}$-Lösung versetzt und $3 \mathrm{mal} \mathrm{mit} 5 \mathrm{ml}$ Chloroform extrahiert. Die organische Phase wird mit $\mathrm{Na}_{2} \mathrm{SO}_{4}$ getrocknet und eingedampft. Zur Gaschromatographie wird in Ethylacetat/Eisessig $(100 \mathrm{ml}+1 \mathrm{ml})$ aufgenommen. Der Nachweis erfolgt durch Injektion von je $2 \mu \mathrm{l}$ des Extraktes an 3 verschiedenen Säulen und Vergleich der relativen Retentionszeiten (Tab. 1). Die Zuordnung wird überprüft durch gleichzeitige Injektion des Extraktes und der entsprechenden Reinsubstanz. Zur quantitativen Bestimmung werden 3 verschiedène Mengen der entsprechenden Standardlösung im Konzentrationsbereich der Pिrobe eingespritżt. Mit Hilfe der Regressionsgeraden aus den Peakhöhen der Kalibrierlösung oder graphisch wird die Konzentration in der Probe ermittelt.

Bei der zusätzlichen dünnschichtchromatographischen Reinigung der Extrakte im System Ethylacetat/Methanol/Ammoniak $(250 \mathrm{~g} / \mathrm{kg})(170 \mathrm{ml}+20 \mathrm{ml}+10 \mathrm{ml})$ erfolgt die Lokalisation durch UV-Absorption (Carbromal, Glutethimid, Methaqualon, Pyrithyldion), durch Ansprïhen mit Kaliumpermanganatlösung $10 \mathrm{~g} / 1$ dest. Wasser - (2,2-Diethylallylacetamid, Methy prylon) oder Quecksilber(I)nitratlösung - $10 \mathrm{~g} / 1$ dest. Wasser -

(Ethinamat) (Tab. 2). Die angegebenen Detektionsmittel dienen zur Lokalisation des entsprechenden Standards, sie werden nicht zum Nachweis der Hypnotica in den Serum-Extrakten benutzt.

Tab. 1. Relative Retentionszeit von nichtbarbiturathaltigen Hypnotica bezogen auf Hexobarbital.

\begin{tabular}{lcll}
\hline Substanz & SP 2250 DA & CDMS & OV-101 \\
\hline Carbromal & n. n. & n. n. & 0,27 \\
2,2-Diethylallylacetamid & 0,09 & 0,06 & 0,12 \\
Ethinamat & 0,15 & 0,14 & 0,16 \\
Glutethimid & 1,03 & 1,00 & 0,96 \\
Methaqualon & 3,63 & 2,07 & 2,41 \\
Methyprylon & 0,35 & 0,28 & 0,28 \\
Pyrithyldion & 0,37 & 0,50 & 0,28 \\
\hline
\end{tabular}

n. n.: Unter den angegebenen gaschromatographischen Bedingungen nicht nachweisbar.

Tab. 2. $R_{F}$-Werte verschiedener nichtbarbiturathaltiger Schlafmittel nach dünnschichtchromatographischer Auftrennung auf Kieselgel-60 beschichteten Aluminiumfolien im Laufmittelsystem Ethylacetat/Methanol/Ammoniak $(250 \mathrm{~g} / \mathrm{kg})(170 \mathrm{ml}+20 \mathrm{ml}+10 \mathrm{ml})$.

\begin{tabular}{lll}
\hline Substanz & $\mathbf{R}_{\mathbf{F}}$-Wert & Nachweis \\
\hline Carbromal & 0,64 & UV-Absorption \\
2,2-Diethylallylacetamid & 0,60 & KMnO $_{4}$-Lösung \\
Ethinamat & 0,66 & HgNO $_{3}$-Lösung \\
Glutethimid & 0,69 & UV-Absorption \\
Methaqualon & 0,70 & UV-Absorption \\
Methyprylon & 0,59 & KMnO \\
Pyrithyldion & 0,58 & UV-Absorption \\
\hline
\end{tabular}

\section{Ergebnisse}

Präzision

$2 \mathrm{ml}$ eines Serums, das keine Hypnotica enthält, werden mit $20 \mu \mathrm{g}$ eines Schlafmittels, sowie mit $0,85 \mathrm{nmol}$ $(200 \mathrm{ng})$ Hexobarbital und $740 . \mathrm{Bq}(20 \mathrm{nCi})\left[2:{ }^{14} \mathrm{C}\right] \mathrm{He}$ - 
Tab. 3. Gaschromatographische Bestimmung von nich tbarbiturathaltigen Schlafmitteln im Serum.

Präzision in der Serie unter Berücksichtigung der Wiederfindung von $[2-14 \mathrm{C}]$ Hexobarbital.

\begin{tabular}{lllll}
\hline Substanz & $\begin{array}{l}\text { Anzahl } \\
\text { der } \\
\text { Ana- } \\
\text { lysen }\end{array}$ & $\begin{array}{l}\text { Soll- } \\
\text { wert }\end{array}$ & $\begin{array}{l}\text { Mittel- } \\
\text { wert }\end{array}$ & $\begin{array}{l}\text { Varia- } \\
\text { tions- } \\
\text { koeffi- } \\
\text { zient }\end{array}$ \\
& $\mathrm{n}$ & $(\mu \mathrm{mol} / \mathrm{l})$ & $\begin{array}{l}\overline{\mathbf{x}} \\
(\mu \mathrm{mol} / \mathrm{l})\end{array}$ & $\mathrm{VK}(\%)$ \\
\hline Carbromal & 10 & 42,2 & 41,6 & 2,1 \\
2,2-Diethylallylacetamid & 10 & 64,4 & 50,9 & 8,5 \\
Ethinamat & 9 & 59,8 & 53,8 & 6,3 \\
Glutethimid & 10 & 46,0 & 45,5 & 5,9 \\
Methy prylon & 8 & 54,6 & 52,4 & 6,5 \\
Pyrithyldion & 7 & 59,8 & 54,4 & 2,9 \\
\hline
\end{tabular}

xobarbital aufgestockt. Je $1 \mathrm{ml}$ dieser Probe wird getrennt aufgearbeitet und analysiert. Unter Berücksichtigung der Wiederfindung des zugesetzten radioaktiven Barbiturats werden die in Tabelle 3 dargestellten Werte erhalten. Der Variationskoeffizient schwankt zwischen 2,1 und $8,5 \%$.

\section{Richtigkeit und Spezifität}

\section{Wiederfindung}

Die Wiederfindung der einzelnen Hypnotica ohne Berücksichtigung der Verluste an radioaktivem Barbiturat ist in Tabelle 4 aufgefuihrt. Sie beträgt zwischen 76 und $92 \%$. Die Methaqualonverluste sind im Gegensatz zu reinen Lösungen bei Verwendung von Serum so hoch, daß das angegebene Verfahren für die quantitative Bestimmung dieses Schlafmittels als nicht geeignet anzusehen ist. Der qualitative Nachweis war jedoch in allen Fällen geführt worden, bei denen katamnestisch eine Methaqualoneinnahme erfahren wurde. Die Verluste der übrigen Hypnotica sind für toxikologische Analysen vernachlässigbaṛ klein.

\section{Spezifität}

Wie für die Barbituratbestimmung gezeigt, erhöht auch bei der Bestimmung anderer Hypnotica der Zusatz von gesăttigter Ammoniumsulfatlösung diè Spezifität und
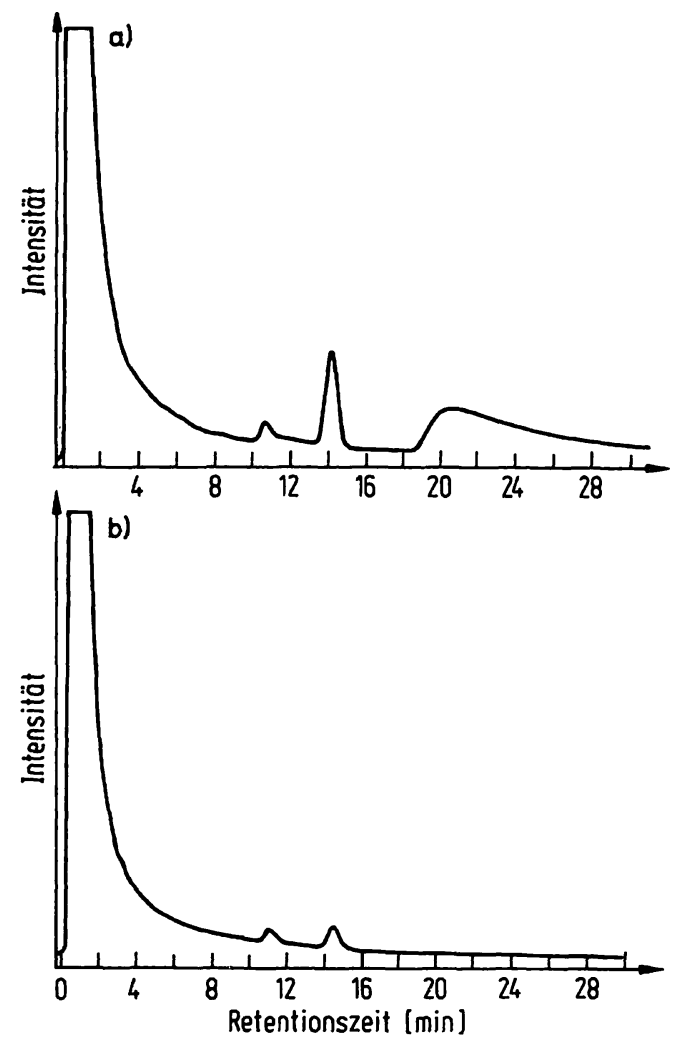

Abb. 1. Gaschromatogramm eines normalen Serums an OV-101 a) nach Extraktion mit Chloroform ohne Zusatz von Ammoniumsulfatlösung.

b) nach Extraktion mit Chloroform und Zusatz von Ammoniumsulfatlösung.

Praktikabilität (Abb. 1). Bei der niedrigen Säulentemperatur, wie sie bei der gaschromatographischen Analyse von Carbromal, 2,2-Diethylallylacetamid und Ethinamat verwendet wird, werden durch den Zusatz an Ammoniumsulfatlösung insbesondere Begleitverunreinigungen entfernt, die aufgrund ihrer langen Retentionszeit folgende Gaschromatogramme stören. Die Abbildungen 2 bis 7 zeigen Gaschromatogramme von Vergiftungen mit den verschiedenen untersuchten Hypnotica. Hämolytische, ikterische, urämische und lipämische Seren können analysiert werden.

Tab. 4. Gaschromatographische Bestimmung von nich tbarbiturathaltigen Schlafmitteln im Serum. Wiederfindung ohne Berücksichtigung der Wiederfindung von $\left[2-{ }^{14} \mathrm{C}\right]$ Hexobarbital.

\begin{tabular}{|c|c|c|c|c|c|}
\hline Substanz & $\begin{array}{l}\text { Anzahl } \\
\text { der Analysen } \\
\text { n }\end{array}$ & $\begin{array}{l}\text { Sollwert } \\
(\mu \mathrm{mol} / 1)\end{array}$ & $\begin{array}{l}\text { Mittelwert } \\
\overline{\mathbf{x}}(\mu \mathrm{mol} / \mathrm{l})\end{array}$ & $\begin{array}{l}\text { Abweichung } \\
\text { vom Sollwert } \\
\Delta \%\end{array}$ & $\begin{array}{l}\text { Variations- } \\
\text { koeffizient } \\
\text { VK (\%) }\end{array}$ \\
\hline $\begin{array}{l}\text { Carbromal } \\
2,2 \div \text { Diethylallylacetämid } \\
\text { Ethinamat } \\
\text { Glutethimid } \\
\text { Methaqualon } \\
\text { Methyprylon } \\
\text { Pyrithyldion }\end{array}$ & $\begin{array}{r}10 \\
10 \\
9 \\
10 \\
10 \\
8 \\
7\end{array}$ & $\begin{array}{l}42,2 \\
64,4 \\
59,8 \\
46,0 \\
39,9 \\
54,6 \\
59,8\end{array}$ & $\begin{array}{r}38,9 \\
49,0 \\
52,5 \\
42,0 \\
7,9 \\
48,9 \\
49,5\end{array}$ & $\begin{array}{l}-7,8 \\
-23,9 \\
-12,2 \\
-8,8 \\
-80,3 \\
-10,4 \\
-17,3\end{array}$ & $\begin{array}{r}3,5 \\
10,0 \\
5,9 \\
6,6 \\
17,1 \\
5,9 \\
1,9\end{array}$ \\
\hline
\end{tabular}

J. Clin. Chem. Clin. Biochem. / Vol. 17, 1979 / No. 3. 


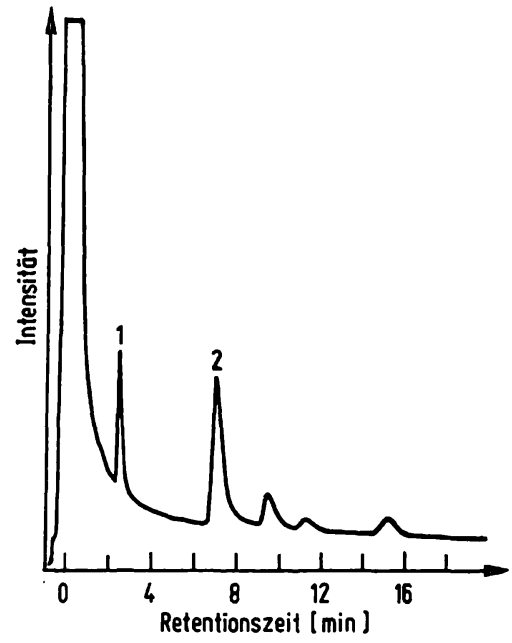

Abb. 2. Gaschromatogramm eines Serums von einer Vergiftung mit Carbromal (1) $(38 \mu \mathrm{mol} / \mathrm{l})$ und Pentobarbital (2) $(40 \mu \mathrm{mol} / 1)$ an $\mathrm{OV}-101$.

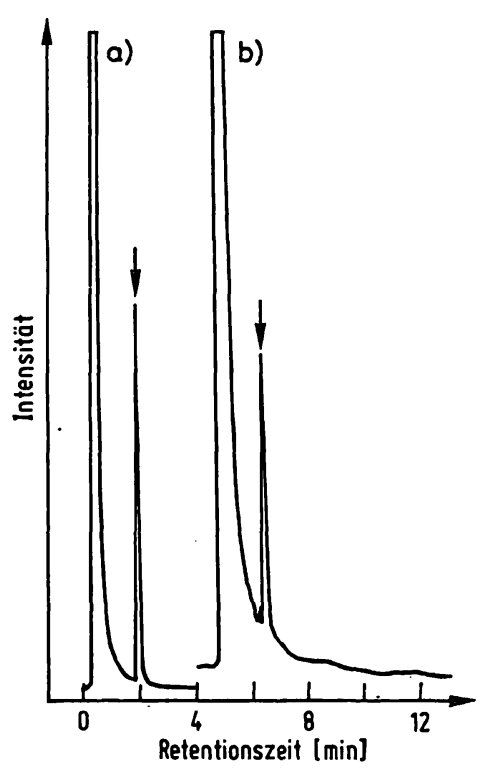

Abb. 3.a) Gaschromatogramm von 1,8 nmol 2,2-Die thylallylacetamid (entsprechend $88 \mu \mathrm{mol} / \mathrm{l}$ ) an OV-101.

b) Gaschromatogramm eines Extraktes aus $0,5 \mathrm{ml}$ Rattenserum - 3 Stunden nach Injektion von 2,2-Diethylallylace tamid gewonnen - an OV-101. Konzentration im Serum $120 \mu \mathrm{mol} / 1 . \downarrow 2,2$-Die thylallylace tamid

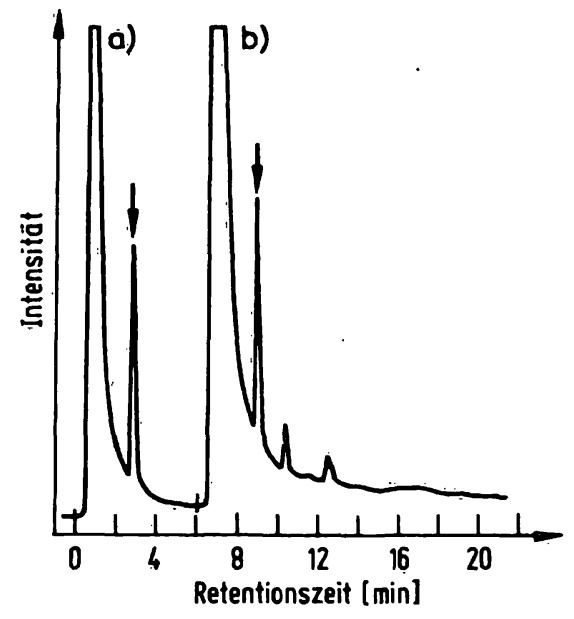

Abb. 4. a) Gaschromatogramm von 0,6 nmol Ethinamat (entsprechend $30 \mu \mathrm{mol} / \mathrm{l}$ ) an OV-101.

b) Gaschromatogramm eines Extraktes aus $0,5 \mathrm{ml}$ Rattenserum - 3 Stunden nach Injektion von Ethinamat gewonnen - an OV-101. Konżentration im Serum $72 \mu \mathrm{mol} / 1 . \downarrow$ Ethinamat

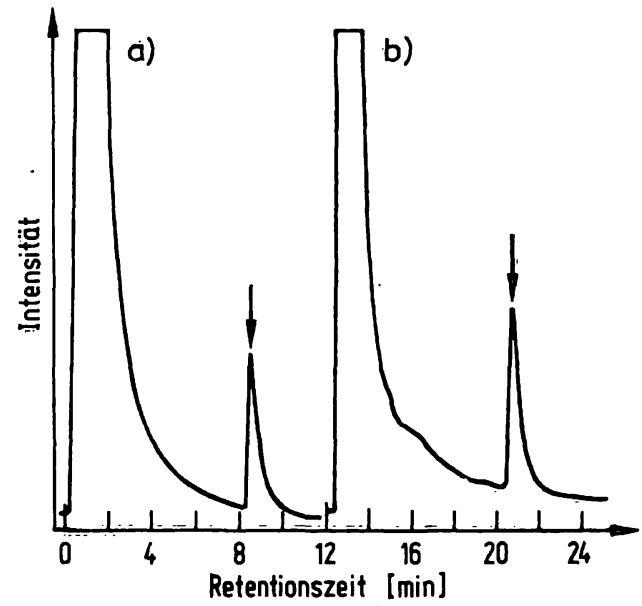

Abb. 5. a) Gaschromatogramm von 0,7 nmol Glutethimid (entsprechend $35 \mu \mathrm{mol} / 1$ ) an SP $2250 \mathrm{DA}$.

b). Gaschromatogramm eines Extraktes aus $0,5 \mathrm{ml}$ Rattenserum - 1 Stunde nach Injektion von Glutethimid gewonnen - an SP 2250 DA. Konzentration im Serum $74 \mu \mathrm{mol} / \mathrm{l}$. $\downarrow$ Glute thimid
Die Analyse von Sera von Menschen oder Tieren (WistarRatten), die mit Schlafmitteln vergiftet waren, vor und nach dünnschichtchromatographischer Reinigung der Extrakte ergeben keine toxikologisch bedeutsamen Unterschiede (Tab. 5).

In manchen Serumextrakten beobachtet man jedoch an SP 2250 DA einen Peak, der eine „Glutethimidkonzentration" von 4,6 bis $9,2 \mu \mathrm{mol} / 1$ vortäuscht. An OV-101 dürften einige Metabolite des Glutethimids nicht abgetrennt und mitbestimmt werden. Wegen der sehr ähnlichen Retentionszeiten an den drei verwendeten stationären Phasen ist eine quantitative Bestimmung von Glutethimid neben Hexobarbital nur schlecht mọglich.

Wegen der Häufigkeit von Mischintoxikationen wurdēn etwa 100 verschiedene Arzneimittelspezialitäten gas- . chromàtographisch analysiert (1): Eine bedeutsame Störung erfährt die Carbromalbestimmung durch 


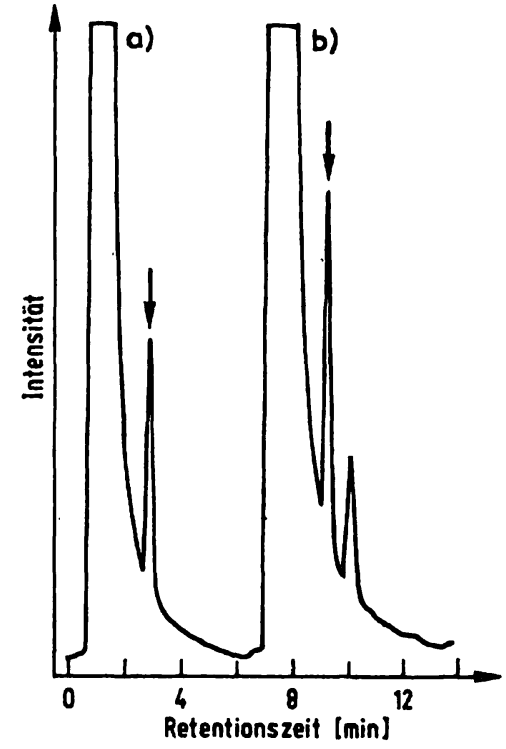

Abb. 6. a) Gaschromatogramm von $1,1 \mathrm{nmol}$ Methyprylon (entsprechend $55 \mu \mathrm{mol} / \mathrm{l}$ ) an CDMS.

b) Gaschromatogramm eines Extraktes aus $0,5 \mathrm{ml}$ Rattenserum - 3 Stunden nach Injektion von Methyprylon gewonnen - an CDMS. Konzentration im Serum $175 \mu \mathrm{mol} / 1 . \downarrow$ Methyprylon

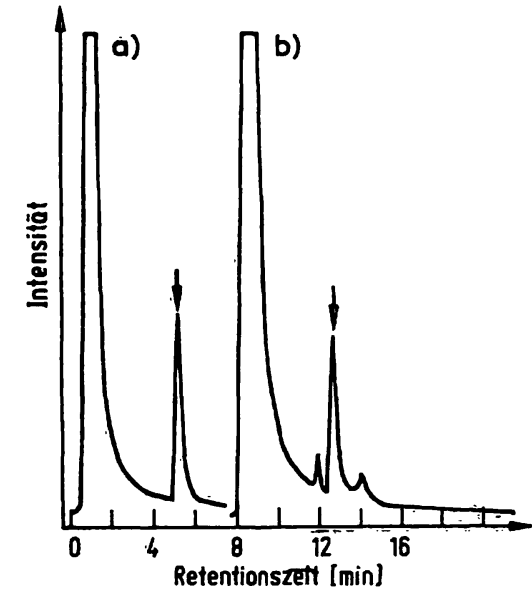

Abb. 7. a) Gaschromatogramm von 1,2 nmol Pyrithyldion (entsprechend $55 \mu \mathrm{mol} / 1$ ) an OV-101.

b) Gaschiomatogramm eines Extraktes aus $0,5 \mathrm{ml}$ Rattenserum - 3 Stunden nach Injektion von Pyrithyldion gewonnen - an OV-101. Konzentration im Serum $108 \mu \mathrm{mol} / \mathrm{l}$. $\downarrow$ Pyrithyldion

Methyprylon und Pyrithyldion an OV-101 $\left(150^{\circ} \mathrm{C}\right.$ Säulentemperatur) wegen der ähnlichen Retentionszeit. Die Unterscheidung zwischen den drei Substanzen gelingt an CDMS bei $230^{\circ} \mathrm{C}$, einer Temperatur, bei der das thermisch labile Carbromal nicht mehr nachweisbar ișt, im Gegensatz̃ zu Methyprylon und Pyrithyldion, die sich an dieser Phase auftrennen lassen. Bei gleichzeitigem Vorkommen von z. B. Methyprylon und Carbromal wird an CDMS eine um den Carbromalanteil
Tab. 5. Gaschromatographische Bestimmung von nichtbarbiturathaltigen Schlafmitteln im Serum.

Vergleich der Analysenergebnisse echter Proben: Messung vor und nach Dünnschichtchromatographie an verschiedenen stationären Phasen unter Berïcksichtigung der Wiederfindung von $\left[2-{ }^{14} \mathrm{C}\right]$ Hexobarbital.

\begin{tabular}{|c|c|c|c|c|}
\hline \multirow{3}{*}{ Substanz } & \multicolumn{2}{|c|}{$\begin{array}{l}\text { vor Dünnschicht- } \\
\text { chromatographie }\end{array}$} & \multicolumn{2}{|c|}{$\begin{array}{l}\text { nach Dünnschicht- } \\
\text { chromatographic }\end{array}$} \\
\hline & $\begin{array}{l}\text { stat. } \\
\text { Phase }\end{array}$ & $\begin{array}{l}\text { stat. } \\
\text { Phasc }\end{array}$ & $\begin{array}{l}\text { stat. } \\
\text { Phase }\end{array}$ & $\begin{array}{l}\text { stat. } \\
\text { Phase }\end{array}$ \\
\hline & \multicolumn{2}{|l|}{$\begin{array}{l}\mathrm{OV}-101 \\
(\mu \mathrm{mol} / \mathrm{l})\end{array}$} & \multicolumn{2}{|l|}{$\begin{array}{l}\text { OV-101 } \\
(\mu \mathrm{mol} / 1)\end{array}$} \\
\hline \multirow[t]{2}{*}{ Carbromal } & $\begin{array}{l}214,8 \\
188,6 \\
174,3 \\
164,6 \\
187,3 \\
233,8 \\
302,1 \\
164,6 \\
115,6 \\
140,9 \\
222,8\end{array}$ & & $\begin{array}{l}218,1 \\
214,8 \\
178,1 \\
171,7 \\
181,0 \\
226,2 \\
270,9 \\
173,8 \\
130,0 \\
146,8 \\
238,4\end{array}$ & \\
\hline & OV-101 & & OV-101 & \\
\hline \multirow[t]{2}{*}{$\begin{array}{l}\text { 2,2-Diethyl- } \\
\text { allylacetamid }\end{array}$} & $\begin{array}{r}102,7 \\
82,9 \\
134,6 \\
272,7 \\
184,1\end{array}$ & · & $\begin{array}{r}79,3 \\
86,4 \\
109,8 \\
249,3 \\
157,2\end{array}$ & . \\
\hline & OV-101 & SP $2250 \mathrm{DA}$ & OV-101 & SP $2250 \mathrm{DA}$ \\
\hline \multirow[t]{2}{*}{ Ethinamat } & $\begin{array}{r}299,0 \\
35,9 \\
63,4 \\
116,6\end{array}$ & $\begin{array}{r}333,7 \\
46,7 \\
64,0 \\
127,4\end{array}$ & $\begin{array}{r}269,1 \\
37,1 \\
66,4 \\
138,8\end{array}$ & $\begin{array}{r}286,5 \\
46,1 \\
76,6 \\
153,7\end{array}$ \\
\hline & OV-101 & SP $2250 \mathrm{DA}$ & OV-101 & SP $2250 \mathrm{DA}$ \\
\hline \multirow[t]{2}{*}{ Glutethimid } & $\begin{array}{r}275,2 \\
377,8 \\
85,6\end{array}$ & $\begin{array}{r}269,7 \\
383,3 \\
71,3\end{array}$ & $\begin{array}{r}272,0 \\
379,7 \\
55,7^{-}\end{array}$ & $\begin{array}{r}272,9 \\
394,4 \\
63,5\end{array}$ \\
\hline & CDMS & & CDMS & \\
\hline \multirow[t]{2}{*}{ Methyprylon } & $\begin{array}{l}287,0 \\
277,1 \\
230,8 \\
269,5\end{array}$ & & $\begin{array}{l}281,0 \\
257,5 \\
237,9 \\
241,1\end{array}$ & \\
\hline & OV-101 & SP $2250 \mathrm{DA}$ & OV-101 & SP $2250 \mathrm{DA}$ \\
\hline Pyrithyldion & $\begin{array}{l}547,2 \\
440,8 \\
531,1 \\
276,3\end{array}$ & $\begin{array}{c}568,2 \\
503,6 \\
602,9 \\
314,0\end{array}$ & $\begin{array}{l}570,0 \\
399,5 \\
515,0 \\
299,6\end{array}$ & $\begin{array}{l}626,2 \\
476,1 \\
549,6 \\
316,4\end{array}$ \\
\hline
\end{tabular}

geringere Menge an „Methyprylon“ gefunden. Die Carbromalkonzentration läßt sich in diesem Fall nur angenähert bestimmen. Die Bestimmung von Carbromal neben Pyrithyldion ist an CDMS unter den Bedingungen, die für die Gaschromatographie an OV-101 ange- 
geben sind, durchführbar. Die relative Retentionszeit von Carbromal an CDMS beträgt 0,28 . Die übrigen untersuchten Substanzen stören die Bestimmung der nichtbarbiturathaltigen Hypnotica nicht, wenn zur Identifizierung die drei Gaschromatographiesysteme benutzt werden.

\section{Nachweisgrenze}

Die Nachweisgrenze wird abgeschätzt durch Analyse von Sera, die mit $2 \mathrm{mg} / \mathrm{l}$ des jeweiligen Schlafmittels aufgestockt wurden. Die Nachweisgrenze schwankt zwischen 1,6 und 4,6 $\mu \mathrm{mol} / \mathrm{l}$ (Tab. 6). Verglichen mit den bei Vergiftungen beobachteten Konzentrationen ist die Methode empfindlich genug, um auch bei Mischintoxikationen mit verschiedenen Schlafmitteln den Nachweis und die quantitative Bestimmung zu gewährleisten.

Tab. 6. Gaschromatographische Bestimmung von nichtbarbiturathaltigen Schlafmitteln im Serum.

Nachweisgrenze ( $3 \mathrm{~s}$-Bereich bei Analyse von Sera, die mit $2 \mathrm{mg} / 1$ des jeweiligen Schlafmittels aufgestockt wurden) unter Berücksichtigung der Wiederfindung von $\left[2-{ }^{14} \mathrm{C}\right]$ Hexobarbital.

\begin{tabular}{lcccl}
\hline Substanz & $\begin{array}{l}\text { An- } \\
\text { zahl } \\
\text { der } \\
\text { Ana- } \\
\text { lysen }\end{array}$ & $\begin{array}{l}\text { Soll- } \\
\text { wert }\end{array}$ & $\begin{array}{l}\text { Mittel- } \\
\text { wert }\end{array}$ & $\begin{array}{l}3 \mathrm{~s}-\mathrm{Be}- \\
\text { reich }\end{array}$ \\
& $\mathrm{n}$ & $(\mu \mathrm{mol} / \mathrm{l})$ & $\begin{array}{c}\overline{\mathrm{x}} \\
(\mu \mathrm{mol} / \mathrm{l})\end{array}$ & $(\mu \mathrm{mol} / \mathrm{l})$ \\
\hline & 8 & 8,4 & 8,7 & 2,8 \\
& 10 & 12,9 & 11,3 & 3,2 \\
Carbromal & 8 & 12,0 & 9,8 & 3,2 \\
2,2-Diethylallylacetamid & 9,2 & 12,3 & 4,6 \\
Ethinamat & 10 & 10,9 & 10,1 & 1,6 \\
Glutethimid & 10 & 10,9 & 10,8 & 2,7 \\
Methyprylon & 8 & 12,0 & 10,8 \\
Pyrithyldion & & & & \\
\hline
\end{tabular}

\section{Praktikabilität}

Das angegebene Verfahren erlaubt die Identifikation und quantitative Analyse von barbiturat- und nichtbarbiturathaltigen Schlafmitteln (Carbromal, 2,2-Diethylallylacetamid, Ethinamat, Glutethimid, Methyprylon und Pyrithyldion) im Serum in 1 bis 2 Stunden. Methaqualon wird qualitativ nachgewiesen. Die Durchfürung der Methode ist einfach; die erhaltenen Serumextrakte sind so weitgehend von Begleitverunreinigungen befreit, daß leicht auswertbare Gaschromatogramme erhalten werden.

\section{Diskussion}

Im Jahre 1977 entfielen wahrscheinlich etwa die Hälfte der Hypnoticasuicide in der Bundesrepublik Deutschland auf Carbromal (3). Bisher wurde zur Erkennung einer akuten Vergiftung mit diesem Schlafmittel gewöhnlich die Methode von Kisser (4) eingesetzt. Das Verfahren liefert - je nach Zeitbedarf für die Veraschung - in etwa 3-6 Stunden eine Angabe über die Gesamtmenge an ionisiertem und kovalent gebundenem Brom. Es erlaubt also nach einer langen Analysendauer nur einen Hinweis auf eine Bromureidvergiftung. Die wichtige Unterscheidung zwischen einer akuten und chronischen Intoxikation gelingt nur unbefriedigend durch eine zusätzliche dünnschichtchromatographische Bestimmung (5), da neben einer akuten auch häufig eine chronische Vergiftung vorliegt. Einen gewissen Fortschritt mag die Methode von Post \& Faber (6) darstellen. Die Bewährung dieses Verfahrens, das Carbromal und 2-Ethylbutyrylharnstoff gemeinsam durch Extrapolation nach UV-Spektrophotometrie bestimmt, steht noch aus. Wir haben etwa gleichzeitig wie Vohland (7) unser gaschromatographisches Verfahren zur Bestimmung von Carbromal entwickelt. Im Gegensatz zur Methode von Vohland (7) erlaubt die beschrièbene Methode nicht nur eine spezifische, quantitative Bestimmung von Carbromal, sondern auch von anderen barbiturat- und nichtbarbiturathaltigen Hypnotica; durch zusätzliche gaschromatographische Analyse an CDMS bei $220^{\circ} \mathrm{C}$ vermeiden wir eine Verwechșlung des Carbromals mit Methyprylon und Pyrithyldion.

Bei Analyse von echten Proben einer Methyprylonvergiftung wurde bei Gaschromatographie an SP 2250 DA im Gegensatz zu aufgestockten Proben ein Metabolit gefunden, der nicht vollständig von der Aụsgangssubstanz abgetrennt wurde. Aufgestockte Sera - und damit auch entsprechende Kontrollsera - eignen sich also nur bedingt zur Qualitätskontrolle von toxikologischen Analysen.

In das Untersuchungsspektrum wurde 2,2-Diethylallylacetamid aufgenommen, da es nach Einfuihrung der Rezeptpflicht für Bromureide das einzige nicht verschreibungspflichtige, chemisch definierte Hypnoticum war, was eine erhebliche Zunahme der toxikologischen Bedeutung erwarten ließ. In der Zwischenzeit hat sich diese Befürchtung erfült (8) und zur Einführung der Verschreibungspflicht (ab 1. 7. 1978) geführt. Eine gaschromatographische Methóde zu seiner Bestimmung wurde bisher nur von Brinkschulte (9) veröffentlicht, ohne Angaben zur Qualitätskontrolle. Die Verwendung eines besser als $\left[2-{ }^{14} \mathrm{C}\right] \mathrm{Hexobarbital}$ geeigneten inneren Standards sollte zu zuverlässigerēn Ergebnissen führen.

Methaqualon besitzt zur Zeit in erster Linie Bedeutung in der „Drogenszene“ (10). Die hypnotische Wirkung ist vom Anwender in Kauf zu nehmen. Mit einem Rückgang des Drogenabusus dürfte auch der Methaqualonmißbrauch abnehmen. Wir schließen an einen qualitativen Nachweis mit dem beschriebenen Verfahren eine quantitative Bestimmung nach Chin \& Fastlich (11) an. Methoden zur gleichzeitigen Bestimmung von Methaqualon neben Barbituraten und Carbromal sind nicht bekannt.

Die beschriebene Methode, die innerhalb von 1 bis 2 Stunden die Identifikation und quantitative Bestimmung von 18 Barbituraten, Carbrómal, 2,2-Diethylallyl- 


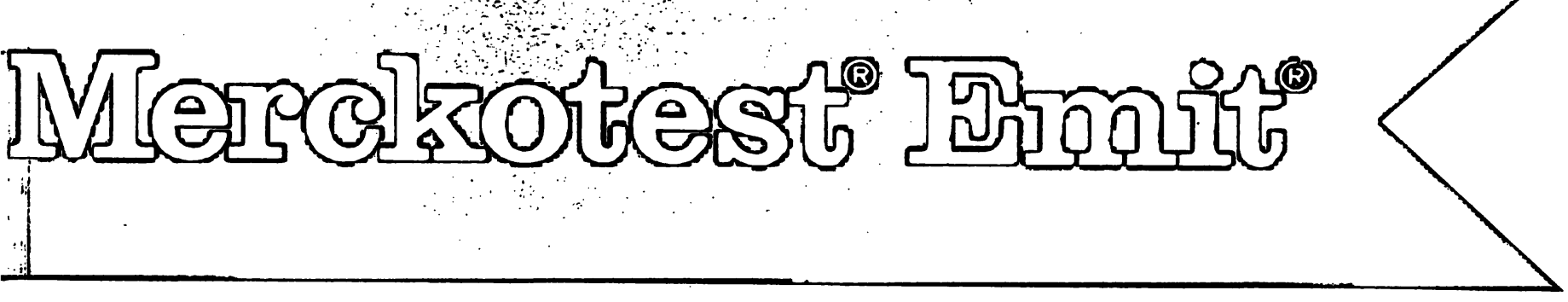

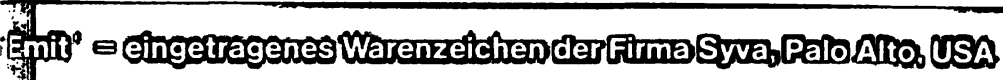

ur Bestimmungoon

ôttepfleptikg

Aur Besthmunguon

irzutuksamensubstanzen

umschifldortsendiagnostik

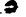

zur Sicherung

ôpethaller Theophyllin=Spiegel

Zum Nachwets bzw:

zurs Sentqua intitativen Bestimmung: inilibreuchilichvenwendeter Arzuefinftrel

\section{Nierekrest Din}

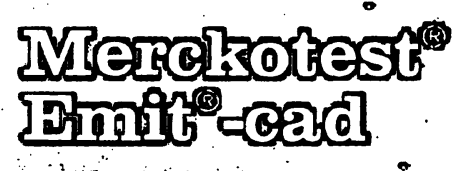

$\$$

iNTerelkotest ,..Thyoxts

Dmathoth

Nerolsotest?

Đา

NTERTotest? Drmitio $=$ dâ
Garbamazepin

Ethosuxtentid

Phenobarbital

Fhenytoín

Petimiton

Digoxth

네dGcain

Theophyilin

Amphetamine

Barbiturate

BesnzodlazepthMetabolite

Cocatnometabolite

Methadorn

Qpiate aed a entiepplieptiondiug

cad E cardibactive drug

tfg 1 thyrofto function group

aad a antiasthmatic drirg

dau a drug abuse urithe
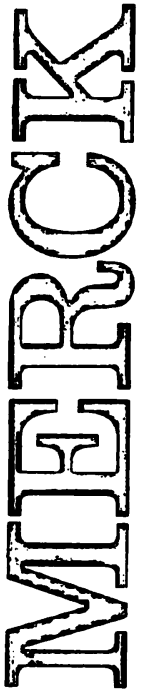

6

(3)

$\circ$

5

¿2

0

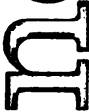

80

60

。각

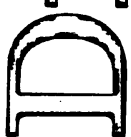

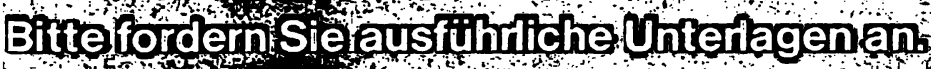




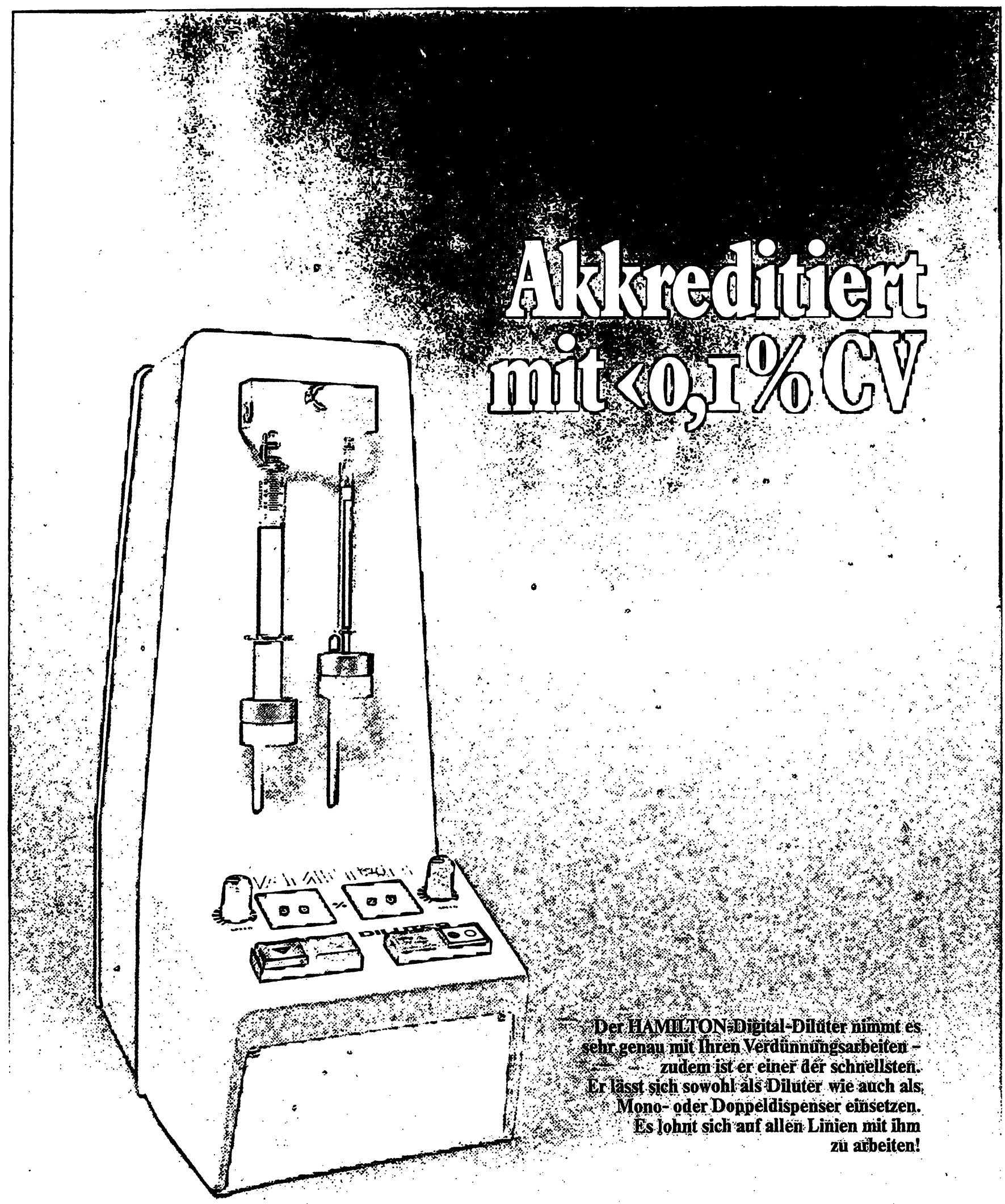

Verlangen Sie ausfựhrliche Unterlagen oder eine Demonstration in Ihrem Labor. Bei Hamilton Bonaduz AG, CH-7402 Bonaduz, Schweiz, oder direkt bei unserer Niederlassung in Deutschland.

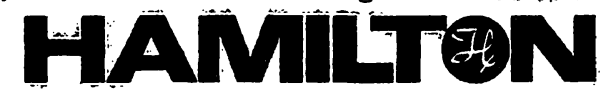


acetamid, Ethinamat, Glutethimid, Methyprylon und Pyrithyldion erlaubt bei qualitativem Nachweis von Methaqualon ist - gemessen am Zeitbedarf und dem Spektrum der erfaßten Substanzen - eine insbesondere den Verhältnissen in der Bundesrepublik Deutschland gut entsprechende Methode.

\section{Literatur}

1. Külpmann, W. R. (1979), diese Z., 17, 89-96.

2. Myschetzky, A. (1971), in Acute barbiturate poisoning (Matthew, H. ed.). Excerpta Medica, Amsterdam, S. 1.

3. v. Clarmann, M. (1977), Intensivbehdlg. 2, 1-6.

4. Kisser, W. (1967), Arch. Toxikol. 22, 404-409.

5. Käferstein, H., Detmer, J. \& Sticht, G. (1974), diese Z. 12, 178-179.

6. Post, D. \& Faber, M. (1976), Klin. Wochenschr. 54, 697698.

\section{Danksagung}

Herrn $K$. Petry und Herm $H$. Lent danke ich für ihre zuverlässige Mitarbeit bei der Durchführung der Bestimmungen.

7. Vohland, H. W., Hadisoemarto, S. \& Wanke, B. (1976), Arch. Toxicol. 36, 31-42.

8. Külpmann, W. R. (1978), Z. Rechtsmed., im Druck.

9. Brinkschulte, M. (1975), Dissertation Tübingen.

10. Stille, G. (1976), Dtsch. Ärztebl. 73, 959-962.

11. Chin, D. \& Fastlich, E. (1974), Clin. Chem. 20, 1382.

Priv. Doz. Dr. W. R. Külpmann Institut $\mathrm{f}$. Klin. Chemie K.-Wiechert-Allee 9 3000 Hannover 61 
